\title{
Thermally induced instability of a doubly quantized vortex in a Bose-Einstein condensate
}

\author{
Krzysztof Gawryluk, ${ }^{1}$ Mirosław Brewczyk, ${ }^{1}$ and Kazimierz Rzążewski ${ }^{2}$ \\ ${ }^{1}$ Instytut Fizyki Teoretycznej, Uniwersytet w Biatymstoku, ulica Lipowa 41, 15-424 Biatystok, Poland \\ ${ }^{2}$ Centrum Fizyki Teoretycznej PAN, Aleja Lotników 32/46, 02-668 Warsaw, Poland
}

(Dated: July 6, 2021)

\begin{abstract}
We study the instability of a doubly quantized vortex topologically imprinted on ${ }^{23} \mathrm{Na}$ condensate, as reported in recent experiment [Phys. Rev. Lett. 93, 160406 (2004)]. We have performed numerical simulations using three-dimensional Gross-Pitaevskii equation with classical thermal noise. Splitting of a doubly quantized vortex turns out to be a process that is very sensitive to the presence of thermal atoms. We observe that even very small thermal fluctuations, corresponding to 10 to $15 \%$ of thermal atoms, cause the decay of doubly quantized vortex into two singly quantized vortices in tens of milliseconds. As in the experiment, the lifetime of doubly quantized vortex is a monotonic function of the interaction strength.
\end{abstract}

Experimental studies of vortices in Bose-Einstein condensates have revealed their peculiar properties related to the quantized circulation [1], originally predicted by Onsager and Feynman in the context of rotating superfluid ${ }^{4} \mathrm{He}$ [2] and further explored in Refs. [3] and [4]. The quantization of the circulation is a manifestation of the existence of a macroscopic wave function. Direct $2 \pi$ change of the phase of the condensate wave function when going around the vortex core was experimentally demonstrated by using the interferometric technique [5]. The quantized vortex can not just disappear, it can leave the condensate or annihilate with a vortex having the opposite circulation. There exists another route for vortices that have multiple topological charge, they can also split into several vortices having smaller charges decreasing in such a way the energy of the system.

In recent experiments 6, 7] doubly quantized vortices have been imprinted in Bose-Einstein condensate of ${ }^{23} \mathrm{Na}$ atoms. A novel approach allowing to create vortices with multiple topological charge was implemented [8]. In this new method, as opposed to dynamical phaseimprinting techniques like rotating the atomic cloud in an anisotropic trap or stirring the condensate with the help of a laser beam, vortices are generated by adiabatically reversing the magnetic bias field along the trap axis. This topological phase imprinting technique leads to vortices displaying winding numbers 2 or 4 depending on the hyperfine state the sodium condensate was prepared in $(\mid 1,-1>$ and $\mid 2,+2>$, respectively) $[\underline{6} \mid$. In Ref. 7] the authors investigate the evolution of doubly quantized vortices by using a tomographic imaging method [9]. They observe the decay of doubly quantized vortex into singly quantized vortices and suggest the possible explanation of this splitting as being a result of dynamical instability, however, not specifying the character of the perturbation seeding the instability.

Existing theoretical explanation of decay of doubly quantized vortices involves the analysis of stability of the vortex at zero temperature in terms of eigenmode spectrum of the Bogoliubov equations in two- and threedimensional geometry as well as the numerical solution of the Gross-Pitaevskii equation in slightly anisotropic trap
10]. Two-dimensional Bogoliubov eigenvalue spectrum shows complex frequencies for certain values of the interaction strength [10, 11]. In fact, quasiperiodic behavior is found - the stability windows are followed by the instability regions. Neither the anisotropy of the trap nor the phenomenologically introduced dissipation is able to force the decay of doubly quantized vortex when there are no complex eigenvalues (i.e., the stability window conditions are fulfilled) [10]. However, in the experiment the monotonic increase of the lifetime of the vortex with the strength of the nonlinearity is observed 7]. It turns out that such behavior is attributed to the three-dimensional geometry of the system and its origin could be due to the presence of trap anisotropy (as suggested in Ref. [10]) or the thermal noise (as claimed by this Letter).

In this Letter we show that only thermal fluctuations, not other disturbances (as, for example, due to the confinement anisotropy), lead to the decay times comparable with that reported in the experiment 7]. To this end, we have performed numerical simulations using the three-dimensional Gross-Pitaevskii equation in the version described in Ref. 12], i.e., with a classical thermal noise. We find that even extremely small thermal fluctuations dramatically accelerate the decay of doubly quantized vortex into two singly quantized vortices. Increasing the number of thermal (uncondensed) atoms already to 10 to $15 \%$ reduces the lifetime of the vortex below $100 \mathrm{~ms}$. Therefore, we argue that although the authors of Ref. 7] report that the experiment is performed under the condition of no discernible thermal atoms presence, the decay of the vortex is triggered by thermal rather than quantum fluctuations.

We describe the system of degenerate bosonic atoms in terms of the classical field that is the complex function representing all atoms not only those within the condensed faction. At zero temperature all atoms are condensed and the classical field becomes the condensate wave function which satisfies the Gross-Pitaevskii equation. However, as it was argued in Ref. [12], the same equation is fulfilled by the classical field at finite temperatures, although in this case it must be interpreted in a different way. The high energy solution of the 
Gross-Pitaevskii equation describes both the condensed and thermal atoms. To get correct physical interpretation of the classical field one has to perform averaging over time or space of corresponding single-particle density matrix. The condensate wave function is just the dominantly populated eigenmode of the averaged singleparticle density matrix. Other modes represent thermal atoms.

We start our simulations with the wave function of the cigar-shaped condensate with a vortex imprinted along the symmetry axis. The topological charge of the vortex equals 2 . The trap parameters are the same as in the experiment of Ref. 7], i.e., the radial (axial) trap frequency equals $220 \mathrm{~Hz}(3 \mathrm{~Hz})$. The number of atoms is changed in such a way that the values of the control parameter defined as $a n_{z}$, where $a$ is the scattering length and $n_{z}=\int|\psi(x, y, z=0)|^{2} d x d y$ is the linear atom density along the symmetry axis taken at the center of the trap, varies up to about 14 (see Ref. 7]). To find the condensate wave function with the vortex we solve the Gross-Pitaevskii equation in imaginary time. The numerical code implements an operator splitting technique as described in [13] and works on two-dimensional grid since the phase of the vortex state, $e^{i 2 \phi}$, can be separated off analytically.

Next, we randomly disturb the condensate wave function, i.e., introduce the thermal noise into the classical field. Since the classical field is a complex function we perturbed independently the amplitude and the phase. Both approaches give very similar results. The evolution of the classical field $\psi$ is governed by the time-dependent Gross-Pitaevskii equation (see Ref. [12])

$$
i \hbar \frac{\partial \psi}{\partial t}=\left(-\frac{\hbar^{2}}{2 m} \nabla^{2}+V_{\text {trap }}+g N|\psi|^{2}\right) \psi
$$

where $g=4 \pi \hbar^{2} a / m$. The above equation is solved on a three-dimensional grid using the Fast Fourier Transform split-operator method. We monitor the diagonal part of the single-particle density matrix that is just the density of the classical field.

In Fig. 1 we plot the time needed to split the doubly quantized vortex as a function of the energy pumped into the system while introducing the thermal noise. For each curve (i.e., the value of the interaction strength) the considered energies range from the zero temperature energy to an amount approximately twice larger. We have checked that this corresponds at most to $20 \%$ condensate depletion. The basic observation is that the decay time is extremely sensitive to the presence of the noise. The rise of the energy by less than $1 \%$ (in comparison with the zero temperature energy) already results in a violant decrease of the decay time. For example, it drops from $200 \mathrm{~ms}$ to $87 \mathrm{~ms}$ for $a n_{z}=5.9$ (the case of zero temperature energy per particle equal to $250 \hbar \omega_{z}$ ). Rigorously speaking, at zero temperature and without any disturbances that might initialize the decay (like the trap anisotropy or any deficiencies related to the process of phase imprinting) the lifetime of the vortex should

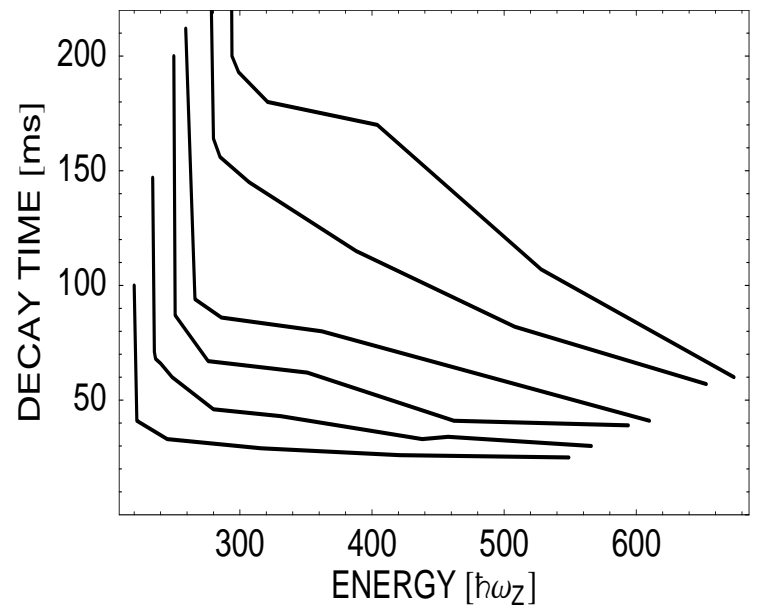

FIG. 1: Decay time of doubly quantized vortex as a function of the energy per particle. The successive curves correspond to different values of the control parameter defined as $a n_{z}$, where $a$ is the scattering length and $n_{z}$ is an axial density at the center of the trap. From top to bottom $a n_{z}$ equals 12.2, $10.0,7.2,5.9,3.9$, and 2.3 , respectively.

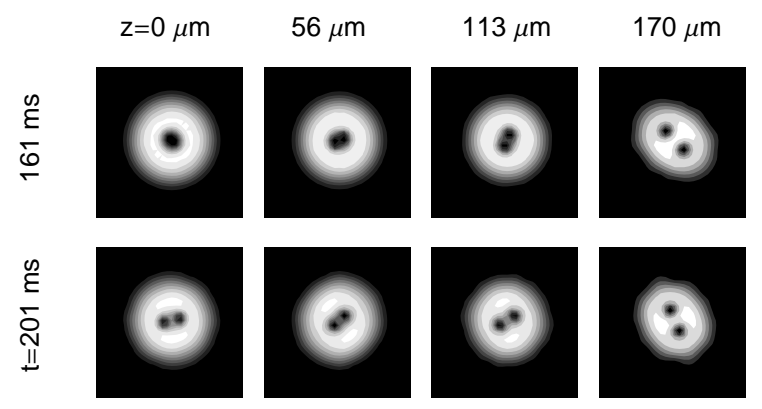

FIG. 2: Density cuts at zero temperature and $a n_{z}=10.0$ for different times showing that even at zero temperature the doubly quantized vortex eventually decays and two singly quantized vortices become disentangled along the whole condensate.

be infinite. Indeed, curves in Fig. 11 show well visible asymptotes when approaching zero temperature energies (although the top-lying end of each curve is a zero temperature decay time that is finite due to the presence of numerical noise). Further increase of the amount of the thermal noise causes additional, although slower, decrease of the decay time. Fig. 1 shows that the decay time becomes comparable with the experimentally measured values (tens of milliseconds) only when the thermal noise at the appropriate level is included in the dynamics.

Even at zero temperature the vortex decays (see Fig. 2). The decay process sets in independently of the density as opposed to what is predicted based on two-dimensional spectral analysis of the Bogoliubov eigenmodes. According to this analysis, the stability windows with respect to the interaction strength exist, the first one appearing approximately for $3<a n_{z}<12$. They are defined by the requirement that all eigenfrequencies of all excitation 
modes are real. Within the stability window no decay of the doubly quantized vortex is expected. Outside the window, i.e., when there is a complex eigenfrequency, the corresponding excitation mode grows exponentially in time resulting in the splitting of the vortex. Adding the third dimension changes the results dramatically as it is shown in Figs. 1 and 2 Splitting of the vortex in three-dimensional condensate need not be a simple and uniform process. In fact, it starts in the region where the local value of $a n_{z}$ is in the two-dimensional instability window (see Ref. [10]). So, the vortex splitting begins at the ends of the condensate (assuming $a n_{z}<12$ ) and propagates towards the center of the trap. In Fig. 1 we plot the lifetime of the doubly quantized vortex understood just as the time needed to split the vortex and disentangle it along the whole condensate.

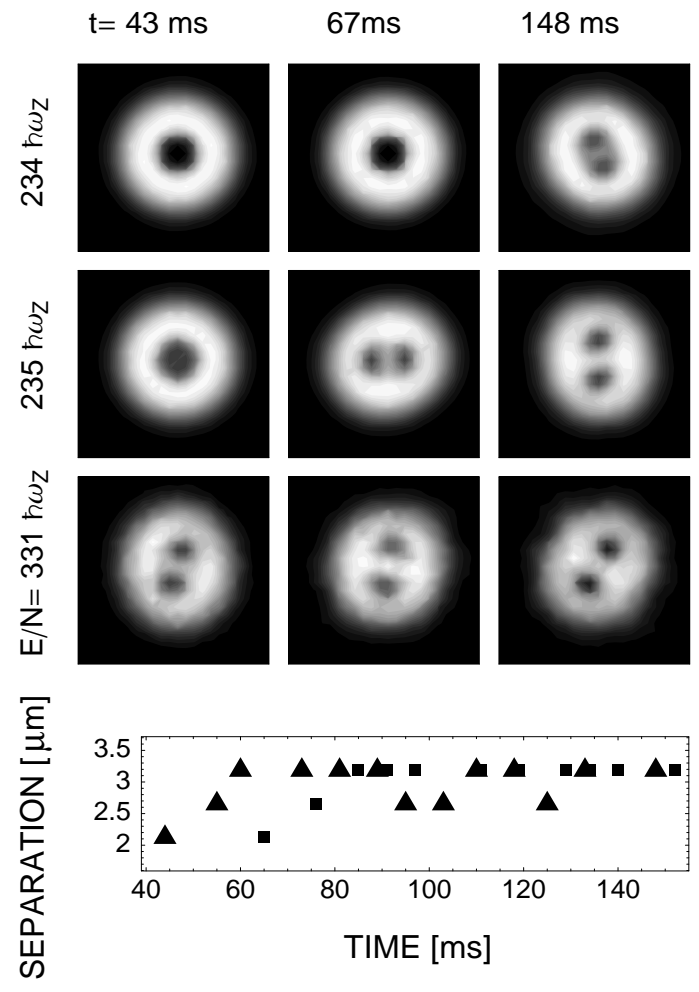

FIG. 3: Tomographic images of the condensate that illustrate the dependence of the decay time on the energy of the system. The interaction strength equals $a n_{z}=3.9$ and the energy per particle is 234,235 , and $331 \hbar \omega_{z}$ from top to bottom, respectively. The lowest panel shows the separation of two singly quantized vortices in the case when $E / N=235 \hbar \omega_{z}$ (squares) and $E / N=331 \hbar \omega_{z}$ (triangles).

In Fig. 3 we compare the decay time of a doubly quantized vortex for different levels of the introduced thermal noise. The pictures are obtained according to the tomographic imaging technique as described in Ref. 7]. In this method, atoms within a $30 \mu \mathrm{m}$ thick central slice of the condensate are transfered to a different hyperfine state and then imaged by using another resonant laser pulse. Following this prescription, each frame in Fig. 3 shows the contour plots of the density integrated axially within the slice of $30 \mu \mathrm{m}$ thickness located at the center of the trap. The first two rows demonstrate how important is the thermal noise. Introducing the thermal noise on a very low level (the energy of the system is increased by less than 1\%) already decreases the time needed to split the vortex by half. Rising the level of the thermal noise causes further lowering of the decay time what is necessary to get an agreement with the experiment. In the bottom panel we plot the separation between two singly quantized vortices as a function of time. The case when the energy of the system is increased by approximately $50 \%$ in comparison with the zero temperature energy is denoted by triangles. Although the population of the condensate is now about $94 \%$, the distance between the two cores $(\sim 3 \mu \mathrm{m})$ almost does not change over the period of $90 \mathrm{~ms}$.

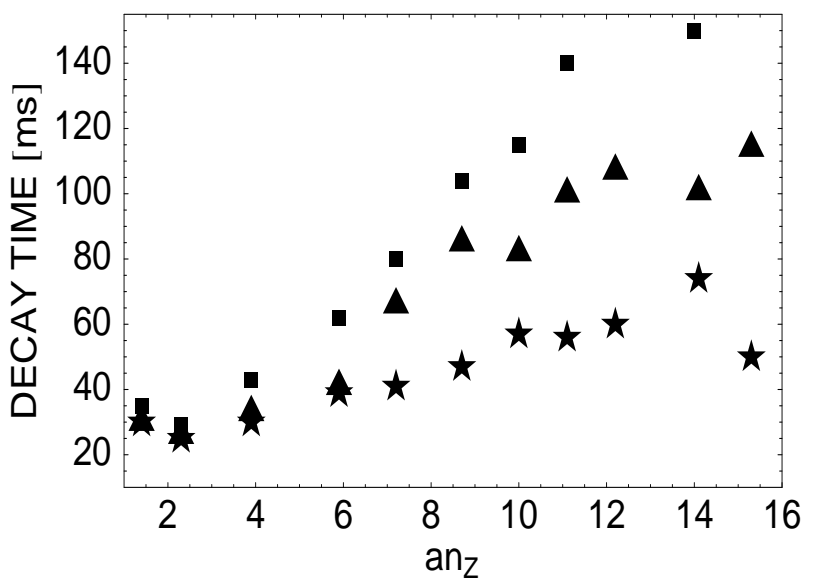

FIG. 4: Decay time of doubly quantized vortex as a function of the density. Three sets of points correspond to different values of the energy brought in the system while introducing the thermal noise. From top to bottom the relative increase of the energy equals $40 \%$ (squares), $85 \%$ (triangles), and $135 \%$ (stars). The condensate depletion amounts to $6 \%, 13 \%$, and $20 \%$, respectively.

Finally, in Fig. 4 we present the data regarding the time of the decay of doubly quantized vortices which are intended to reproduce the main result of the experimental work 7]. The authors of Ref. 7] say only that the experiment was performed at the lowest possible temperature. Since the temperature of the system is determined based on the expansion of the thermal cloud, this statement puts on, in fact, the constraint on the number of thermal atoms. To our knowledge the presence of less than $15 \%$ of thermal atoms can not be detected by using currently available experimental techniques. Therefore, in Fig. 4 we plot three sets of data, each of them corresponding to different level of thermal noise. More precisely, the relative increase of the energy of the system is constant for each data set. It is clear from Fig. 4 that the decay time gets larger when the interaction strength increases what remains in agreement with the experiment. For larger 
density, however, the decay time is getting saturated.

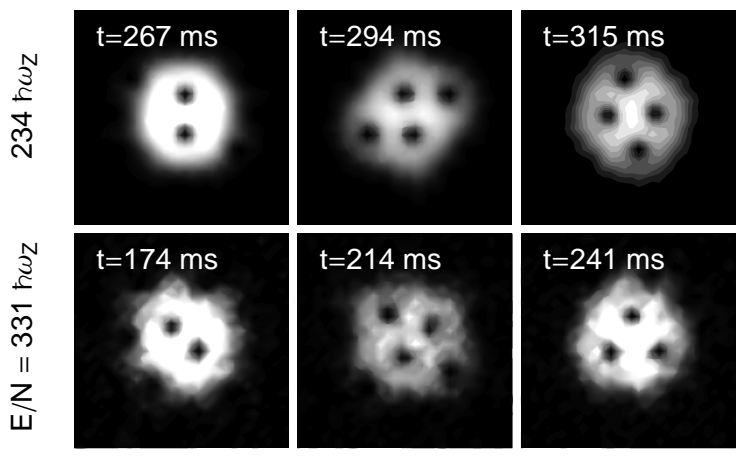

FIG. 5: Density cuts along the radial plane $z=0$ at zero (the first row) and finite (the bottom row) temperatures for the interaction strength $a n_{z}=3.9$.

To answer the question whether other sources of instability are able to reproduce the experimental results of Ref. [7] we investigated the influence of the trap anisotropy (as high as 2.3\% according to [14]) caused by the gravitational sag as well as imprinting deficiencies related to the rapid change of the magnetic field producing the vortex and resulting in radial squeeze and vertical kick of the condensate 7, 14]. We checked that the trap anisotropy makes the lifetime of a doubly quantized vortex finite but still bigger than the experimental values. For example, for $a n_{z}=10.0$ the decay time equals $170 \mathrm{~ms}$ whereas for $a n_{z}=3.9$ it is $76 \mathrm{~ms}$. Moreover, since the gravitational field do not fluctuate the trap anisotropy can not explain the huge scatter of data in Fig. 3 of Ref. [7]. Shifting the whole condensate off the center of the trap also does not help. It makes the total energy higher by increasing the potential energy not the kinetic one. Therefore the number of uncondensed atoms remains constant and the lifetime of the vortex equals that for unshifted condensate. Finally, we considered the dynamical phase imprinting instead of the topological one. In this case the energy pumped into the system goes to the kinetic energy and results in a production of uncon- densed atoms. The decay time is comparable with the experimental values revealing in this way the basic role of the uncondensed atoms in the process of decaying of doubly quantized vortices.

Another interesting phenomenon is discovered while tracing the dynamics of the splitting and subsequent evolution of two singly quantized vortices for longer times. It turns out that additional vortices enter the condensate. In Fig. 5 the first row corresponds to the case of zero temperature. Here, we observe that two singly quantized vortices (as numerically checked by using the interference technique as described in [15]) enter the condensate at the same time and settle into a lattice (in a rotating frame of reference) realizing the first scenario reported in Ref. 16. At finite temperatures (the last row) the dynamics is reacher. First, two additional vortices simultaneously enter the condensate, later on, however, one vortex is lost and the system ends with the lattice consisting of three vortices.

In conclusion, we have addressed the issue concerning the lifetime of a doubly quantized vortex raised by a recent experiment 7]. We show that the decay of a doubly quantized vortex is driven rather by the thermal fluctuations than other kinds of perturbation (quantum fluctuations since we need 10 to $15 \%$ of thermal atoms to achieve the agreement with experiment [7] or the trap anisotropy). The thermal noise is the only reason, we find, able to decrease the time needed to split the vortex and make it comparable to the experimental values. As a result, the decay time is a monotonic function of the interaction strength. Our stressing of the role of thermal noise does not contradict the statement of the authors of Ref. 7] saying that the experiment was performed at the lowest possible temperature.

\section{Acknowledgments}

We thank M. Gajda for helpful discussions. We acknowledge support by the Polish Ministry of Scientific Research Grant Quantum Information and Quantum Engineering No. PBZ-MIN-008/P03/2003.
[1] M. R. Matthews et al, Phys. Rev. Lett. 83, 2498 (1999); K. W. Madison et al, Phys. Rev. Lett. 84, 806 (2000); J. R. Abo-Shaeer et al, Science 292, 476 (2001).

[2] L. Onsager, Nuovo Cimento 6, 249 (1949); R. P. Feynman, in Progress in Low Temperature Physics, edited by C. J. Gorter (North-Holland, Amsterdam, 1955), Vol. 1.

[3] A. L. Fetter, Phys. Rev. 162, 143 (1967); M. Rasetti and T. Regge, Physica A 80, 217 (1975).

[4] A. Hansen et al, Phys. Rev. Lett. 55, 1431 (1985); J. P. Kottman et al, Phys. Lett. A 242, 99 (1998); V. Penna, Phys. Rev. B 59, 7172 (1999).

[5] F. Chevy et al, Phys. Rev. A 64, 031601(R) (2001).

[6] A. E. Leanhardt et al, Phys. Rev. Lett. 89, 190403
(2002).

[7] Y. Shin et al, Phys. Rev. Lett. 93, 160406 (2004).

[8] T. Isoshima et al, Phys. Rev. A 61, 063610 (2000); S.-I. Ogawa et al, Phys. Rev. A 66, 013617 (2002).

[9] M. R. Andrews et al, Science 275, 637 (1997).

[10] M. Möttönen et al, Phys. Rev. A 68, 023611 (2003).

[11] H. Pu et al, Phys. Rev. A 59, 1533 (1999).

[12] H. Schmidt et al, J. Opt. B 5, S96 (2003).

[13] W. H. Press et al, Numerical Recipes: The Art of Scientific Computing (Cambridge University Press, Cambridge, 1986).

[14] Y. Shin and W. Ketterle (private communication).

[15] E. Dobrek et al, Phys. Rev. A 60, R3381 (1999); G. An- 
drelczyk et al, Phys. Rev. A 64, 043601 (2001).

[16] C. Lobo et al, Phys. Rev. Lett. 92, 020403 (2004). 